\title{
NONLINEAR MODELING OF CARDIOVASCULAR RESPONSE TO EXERCISE
}

\author{
Lu Wang ${ }^{1}$, Steven W. Su${ }^{2,1}$, Gregory S. H. Chan ${ }^{1}$, Branko G. Celler ${ }^{1 *}$, Teddy M. Cheng ${ }^{1}$ \\ and Andrey V. Savkin ${ }^{1}$ \\ ${ }^{1}$ Biomedical System Lab, School of Electrical Engineering \& Telecommunications, Faculty of Engineering \\ University of New South Wales, UNSW Sydney, N.S.W. 2052, Australia \\ *b.celler@unsw.edu.au \\ ${ }^{2}$ Key University Research Centre for Health Technologies, Faculty of Engineering, University of Technology \\ Sydney,PO Box 123 Broadway NSW 2007 Australia
}

Keywords: Cardiovascular system, Nonlinear modelling, Cardiovascular responses to Exercise, Machine learning.

\begin{abstract}
This study experimentally investigates the relationships between central cardiovascular variables and oxygen uptake based on nonlinear analysis and modeling. Ten healthy subjects were studied using cycleergometry exercise tests with constant workloads ranging from 25 Watt to 125 Watt. Breath by breath gas exchange, heart rate, cardiac output, stroke volume and blood pressure were measured at each stage. The modeling results proved that the nonlinear modeling method (Support Vector Regression) outperforms traditional regression method (reducing Estimation Error between 59\% and 80\%, reducing Testing Error between $53 \%$ and $72 \%$ ) and is the ideal approach in the modeling of physiological data, especially with small training data set.
\end{abstract}

\section{INTRODUCTION}

The relationships between central cardiovascular variables and oxygen uptake during steady state of graded exercise have been widely examined by numerous investigators (Allor et al., 2000) (Astrand et al., 1964) (Fairbarn et al., 1994) (Freedman et al., 1955) (Kobayashi et al., 1978) (Reeves et al., 1961) (Richard et al., 2004) (Rowland et al., 1997) (Turley et al., 1997). Most of them investigated the relationship between cardiac output (CO) and oxygen uptake $\left(\dot{V} \mathrm{O}_{2}\right)$ using linear regression methods and found the slope between the two variables to be approximately $5-6$ in normal and athletic subjects (Rowell et al., 1986). Beck et al (Beck et al., 2006) in contrast, investigated this relationship in healthy humans using polynomial regression. Turley (Turley et al., 1997) described both the relationship of stroke volume (SV) and the total peripheral resistance (TPR) to oxygen uptake during steady state of sub-maximal exercise using linear regression. However, from the point view of modeling, the regression methods used by the previous researchers have several limitations. First the empirical risk minimization (ERM) principle used by traditional regression models does not guarantee good generalization performance and may produce models that over-fit the data (Gunn, 1997). Secondly, most of the regression models developed from early research based on a small sample set with limited subjects during three or four exercise intensities. Traditional regression approachs are particularly not recommended for modeling small training sets. Determination of the size of the training set is a main issue to be solved in the modeling performance because the sufficiency and efficiency of the training set is one of the most important factors to be considered.

This study presents a novel machine learning approach, Support Vector Regression (SVR) (Drucker et al., 1997) to model the central cardiovascular response to exercise. SVR, developed by Vapnik and his co-workers in 1995, has been widely applied in forecasting and regression ( $\mathrm{Su}$ et al., 2007) (Su et al., 2005) (Su et al., 2006) (Valerity et al., 2003). The following characteristics of SVR make it an ideal approach in modeling of cardiovascular system. Firstly, SVR avoids the overfitting problem which exists in the traditional modeling approaches. Second, SVR condenses information in the training data and provide a sparse 
representation by using a small number of data points (Girosi, 1998). Thirdly, SVR is insensitive to modeling assumption due to its being a nonparametric model structure. Finally, the SVR model is unique and globally optimal, unlike traditional training which can risk converging to local minima.

The rest of this paper is organized as follows: section 2 describes the experimental design for the data collection. Section 3 applies SVR for modeling the relationships between central cardiovascular variables and oxygen uptake. Finally, some conclusions are drawn in Section 4.

\section{EXPERIMENTAL DESIGN}

\subsection{Subjects}

We studied 12 normal male subjects. They are all active, but do not participate in formal training or organized sports. However, since two of them could not complete 6 minutes of higher level exercise, only the data recorded from 10 subjects (aged $25 \pm 4 \mathrm{yr}$, height $177 \pm 5 \mathrm{~cm}$, body weight $73 \pm 11 \mathrm{~kg}$ ) are used for this study. All the subjects knew the protocol and the potential risks, and had given their informed consent.

\subsection{Experimental Procedure}

All tests were conducted in the afternoon in an airconditioned laboratory with temperature maintained between $23-24{ }^{\circ} \mathrm{C}$. The subjects were studied during rest and a series of exercise in an upright position on an electronically braked cycle ergometer. Exercise was maintained at a constant workload for 6 minutes, followed by a period of rest. The initial exercise level was $25 \mathrm{~W}$ and each successive stint of exercise was increased in $25 \mathrm{~W}$ steps until a workload of $125 \mathrm{~W}$ was reached. The rest periods were increased progressively from 10 to 30 minutes after each stint of exercise. Six minutes of exercise was long enough to approach a steady state since the values of oxygen uptake and the $\mathrm{A}-\mathrm{V}$ oxygen difference had become stable by the 5th and 6th minutes even for near maximum exertion (Reeves et al., 1961).

\subsection{Measurement and Data Processing}

Heart rate was monitored beat by beat using a single lead ECG instrument, while ventilation and pulmonary exchange were measured on a breath by breath basis. Minute ventilation was measured during inspiration using a Turbine Flow Transducer model K520-C521 (Applied Electrochemistry, USA). Pulmonary gas exchange was measured using S-3A and CD-3A gas analyzers (Applied Electrochemistry, USA). Before each individual exercise test, the turbine flow meter was calibrated using a 3.0 liters calibration syringe. Before and after each test, the gas analyzers were calibrated using reference gases with known $\mathrm{O}_{2}$ and $\mathrm{CO}_{2}$ concentrations. The outputs of the ECG, the flow transducer and the gas analyzers were interfaced to a laptop through an A/D converter (NI DAQ 6062E) with a sampling rate of $500 \mathrm{~Hz}$. Programs were developed in Labview 7.0 for breath by breath determination of pulmonary gas exchange variables but with particular reference to $\dot{V} O_{2}\left(\dot{V} O_{2}\right.$ STPD).

Beat by beat stroke volume and cardiac outputs were measured noninvasively using the ultrasound based device (USCOM, Sydney, Australia) at the ascending aorta. This device has previously been reported to be both accurate and reproducible (Knobloch et al., 2005). In order to keep consistent measurements, all $\mathrm{CO} / \mathrm{SV}$ measurements were conducted by the same person. An oscillometric blood pressure measurement device (CBM-700, Colin, France) was used to measure blood pressure.

The measurement of $\dot{V} O_{2}$ and $\mathrm{HR}$ were conducted during the whole exercise and recovery stage. The static values ( $\dot{V}_{2}$ and HR) were calculated for each workload from data collected in the last minute of the six minute exercise protocol. The measurements of SV, CO and BP (blood pressure) were similarly conducted during the last minute of the six minute exercise for each workload with the additional requirement that subjects keep their upper body as still as possible to minimize artifacts caused by the movement of the chest during exercise. We then, calculated their static values $(\mathrm{CO}$, $\mathrm{SV}$ and BP) based on the measurement in the last minute for each workload.

\subsection{Results}

We found that the percentage changes of cardiovascular variables relative to their rest values more uniform than when absolute values are used. This may be because using relative values diminish the variability between subjects. For example, Figure 1 (a) shows the relationship between the absolute value of mean arterial blood pressure response and the absolute value of oxygen uptake rate for all the ten subjects, while Figure 1 (b) is the percentage change in mean arterial blood pressure relative to its rest value with the percentage change in oxygen uptake rate to its rest value for the ten 
subjects. Obviously, the response in Figure 1 (b) is more consistent and gives clearer trend than that in Figure 1 (a). It is thus reasonable to believe that modeling of cardiovascular responses using relative changes may give more robust results than modeling with the absolute values.

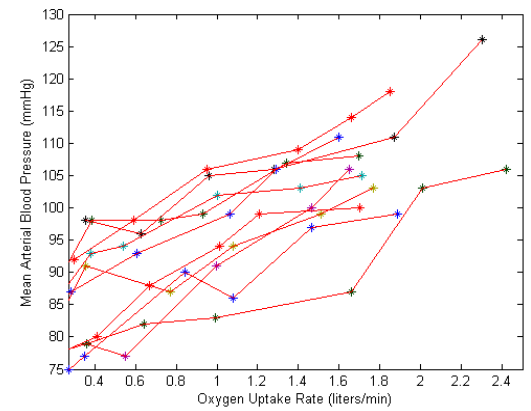

(a). Mean arterial blood pressure response to oxygen uptake rate (in absolute value).

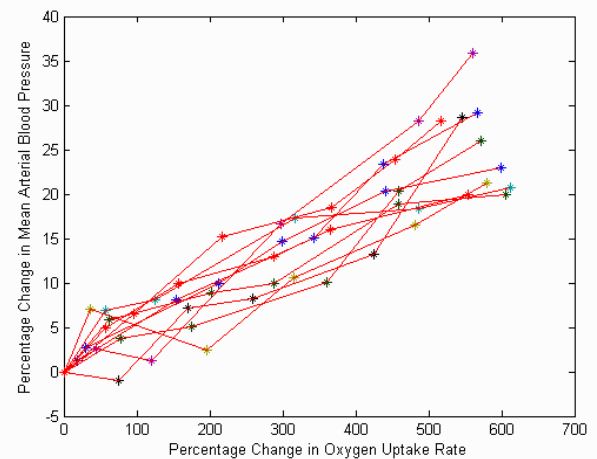

(b). Mean arterial blood pressure response to oxygen uptake rate (in relative value).

Figure 1: Mean arterial blood pressure response to oxygen uptake rate for the ten subjects.

Based on the above finding, we model $\mathrm{CO}, \mathrm{SV}$ and TPR to $\dot{V} O_{2}$ by modeling the percentage changes in $\mathrm{CO}, \mathrm{SV}$ and TPR with respect to their corresponding rest values to percentage change in $\dot{V} \mathrm{O}_{2}$ with respect to its rest value. We use $\mathrm{CO} \%$, $\mathrm{SV} \%, \mathrm{TPR} \%$ and $\dot{\mathrm{VO}}_{2} \%$ to represent their relative values (expressed as percentage), respectively.

\section{APPLICATION OF SVR FOR MODELING}

We selected radial basic function (RBF) kernels for this study, that is $K\left(x, x_{i}\right)=\exp \left(-\frac{\left\|x-x_{i}\right\|^{2}}{2 \sigma^{2}}\right)$ where $\sigma$ is the kernel parameter, $x_{i}$ is the ith input support value and $x$ is the input value.

Detailed discussion about SVR, such as the selection of regularization constant $C$, radius $\varepsilon$ of the tube and kernel function, can be found in (Gunn, 1997) (Vapnik, 1998).

In order to show the effectiveness of SVR, we applied both SVR and traditional linear regression (Least-Square linear regression (LS)) to investigate the relationships between percentage change of cardiovascular variables $(\mathrm{CO} \%, \mathrm{SV} \%$ and $\mathrm{TPR} \%)$ and $\dot{V} \mathrm{O}_{2} \%$.

\subsection{The Relationship between CO\% and $\dot{V} \mathrm{O}_{2} \%$}

\subsubsection{Model Identification}

A SVR model was developed to estimate $\mathrm{CO} \%$ from $\dot{V} \mathrm{O}_{2} \%$ (Table 1 and Figure 2). Although it is widely accepted that there is a linear relationship between cardiac output and oxygen consumption (Allor et al., 2000) (Astrand et al., 1964) (Freedman et al., 1955),

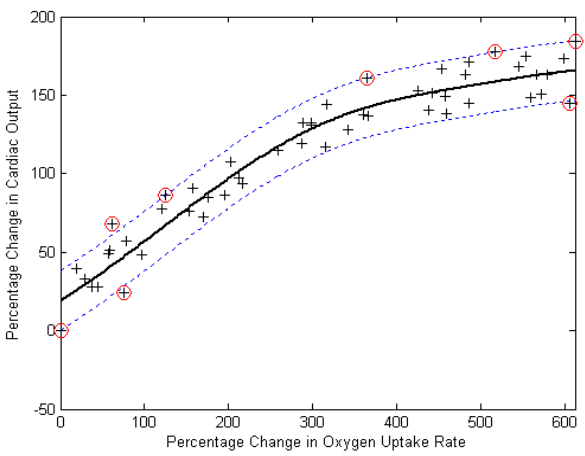

(a). Estimation of percentage change in $\mathrm{CO}$ from percentage change in $\dot{\mathrm{V}} \mathrm{O}_{2}$ using SVR.

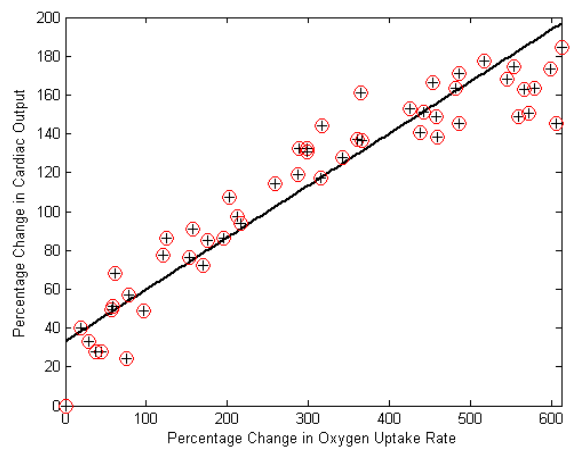

(b). Estimation of percentage change in $\mathrm{CO}$ from percentage change in $\dot{\mathrm{V}} \mathrm{O}_{2}$ using LS.

Figure 2: Comparison of estimation results of $\mathrm{CO} \%$ between using SVR and using LS. 
their relationship can be better described by the nonlinear SVR model in terms of reducing the errors (MSE) from 418 to 171 (Table 2), an improvement of $59 \%$ comparing with that of LS method

The results in Table 1 also show the efficiency of SVR. Unlike traditional regression method where the solution of the model depends on the whole training data points, in SVR, the solution to the problem is only dependent on a subset of training data points which are referred to as support vectors. Using only support vectors, the same solution can be obtained as using all the training data points. SVR uses just $13 \%$ of the total points available to model their nonlinear behavior efficiently.

\subsubsection{Model Validation}

To further evaluate the feasibility of this proposed SVR model, the whole data set is divided into two parts: the first part ( $70 \%$ of the data) is used to design the model and the second part $(30 \%$ of the data) is used to test its performance. Because we do not have large sample of data, we separated the data set into two parts randomly five times. Each time we use $70 \%$ of the data for training and the rest for testing. We established the SVR model with the three design parameters (kernel function, capacity $(C)$ and the radius of insensitivity $(\varepsilon)$ ) based on the training set, and test its goodness on the testing set. In Figure 3, we present the results for one of the 5 tests. As shown in Table 3, the averaged results (MSE) for the 5 times testing for SVR is $245 \pm 15$. However, the averaged error for traditional linear regression is as high as $521 \pm 19$. It indicates that SVR can build more robust models to predict $\mathrm{CO} \%$ from $\dot{V} \mathrm{O}_{2} \%$ using only a small training set. It also demonstrates that SVR can overcome the overfitting problem, even though SVR has more model parameters than the traditional linear regression method.

\subsection{The Relationship between SV\% and $\dot{V}_{2} \%$}

Figure 4 shows the models for estimating SV\%. The SVR model gives more precisely estimation than the LS does and decreases estimation errors (MSE) by $67 \%$ (Table 2).

The testing models are given in Figure 5 and the testing errors are in Table 3. As indicated, the SVR model decreases the testing error by $64 \%$.

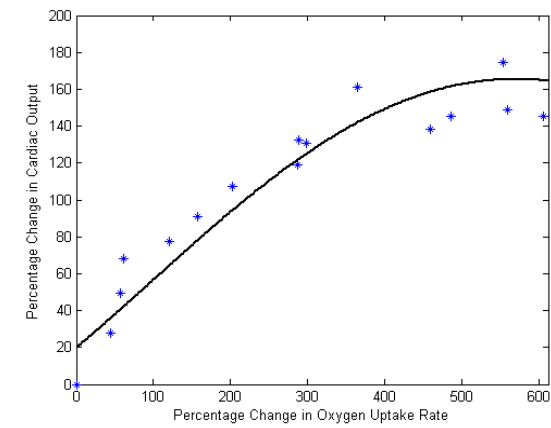

(a). Testing of SVR model.

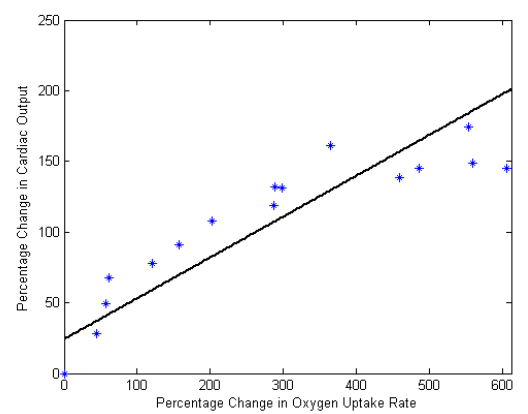

(b). Testing of LS model

Figure 3: Comparison of models of $\mathrm{CO} \%$ against \% change in oxygen uptake using SVR and using LS methods.

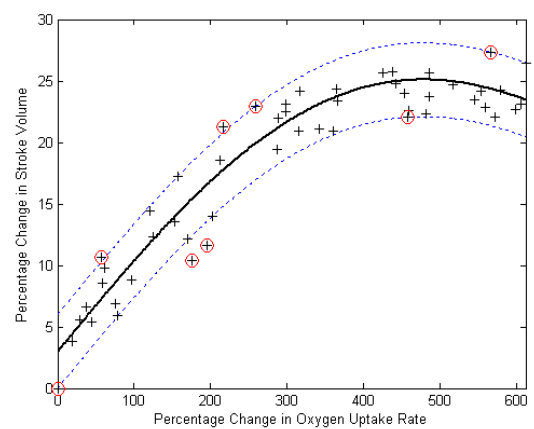

(a). Estimation of percentage change in SV from percentage change in $\dot{V} O_{2}$ using SVR.

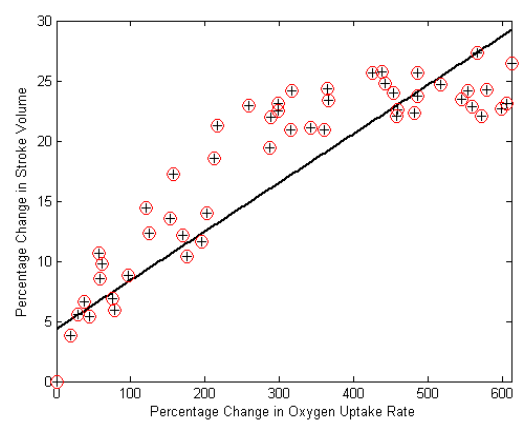

(b). Estimation of percentage change in SV with percentage change in $\dot{\mathrm{V}} \mathrm{O}_{2}$ using linear regression.

Figure 4: Comparison of estimation results for SV\% between using SVR and using LS. 


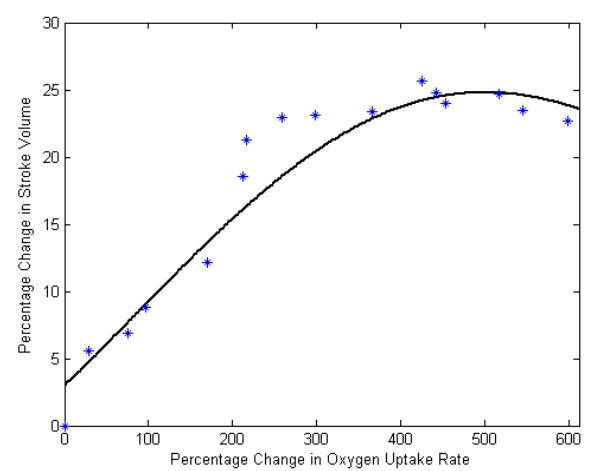

(a). Testing of SVR model.

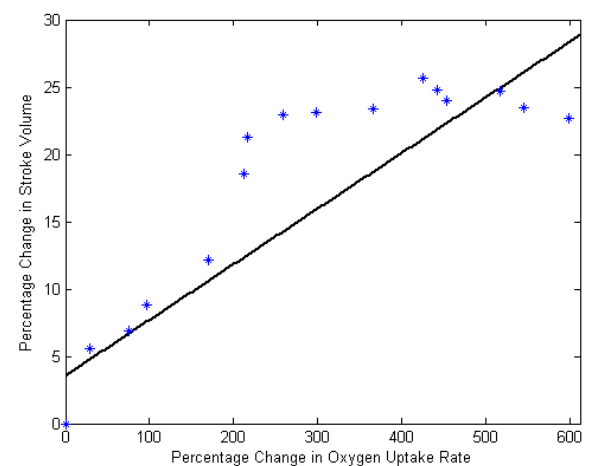

(b). Testing of LS model.

Figure 5: Comparison of the testing results for Stroke Volume using SVR and using traditional linear regression.

\subsection{The Relationship between TPR\% and $\dot{V} \mathrm{O}_{2} \%$}

As shown in Figure 6, the SVR model describes a rapid fall in $\mathrm{TPR} \%$ at low workloads which remains relatively constant even with increasing $\dot{\mathrm{V}} \mathrm{O}_{2} \%$. SVR uses just $13 \%$ (Table 1 ) of the total points to get an efficient nonlinear model. Compared with linear regression, the SVR model decreases MSE from 151 to 30 , an improvement of $80 \%$.

The testing results for this SVR model and the equivalent LS model are given in Figure 7 and Table 3 , respectively. Both of these (Figure 7 and Table 3) demonstrate that SVR outperforms the traditional linear regression method by reducing testing errors significantly, from 130 to 36 .

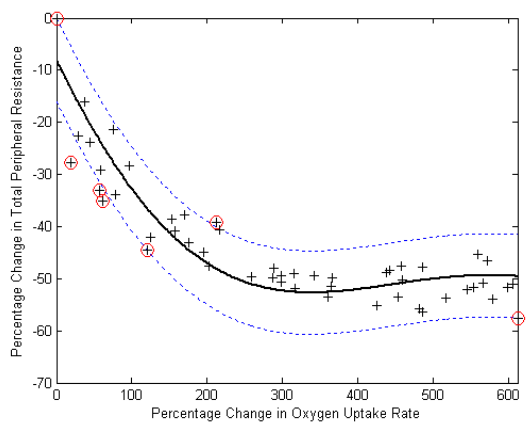

(a). Estimation of percentage change in TPR from percentage change in $\dot{V} O_{2}$ using SVR.

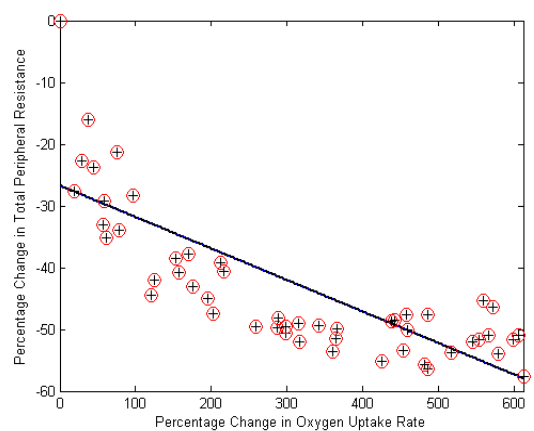

(b). Estimation of percentage change in TPR with percentage change in $\dot{V} O_{2}$ using linear regression.

Figure 6: Comparison of the estimation results of TPR\% between using SVR and LS.

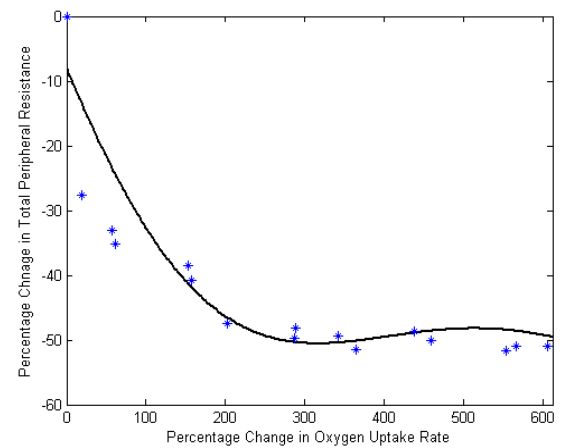

(a). Testing of SVR model.

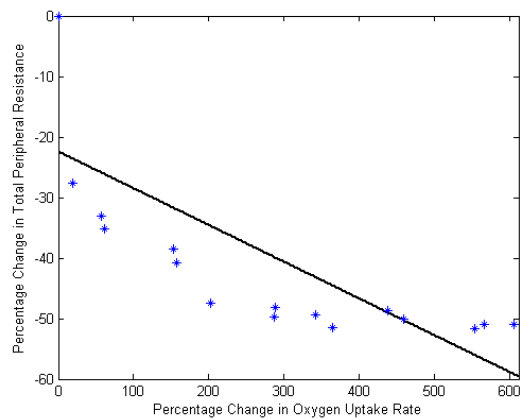

(b). Testing of LS model.

Figure 7: Comparison of the test results of TPR\% against $\%$ change on Oxygen uptake using SVR and using LS. 
Table 1: Fitting data for the model of cardiovascular variables and oxygen uptake rate using SVR.

\begin{tabular}{|c|c|c|c|}
\hline Relation & $\begin{array}{c}\mathrm{CO} \% \mathrm{Vs} \\
\dot{V} O_{2} \%\end{array}$ & $\begin{array}{c}\mathrm{SV} \% \mathrm{Vs} \\
\dot{V} O_{2} \%\end{array}$ & $\begin{array}{c}\mathrm{TPR} \% \\
\mathrm{Vs} \\
\dot{V} O_{2} \%\end{array}$ \\
\hline Kernel & $\mathrm{RBF}$ & $\mathrm{RBF}$ & $\mathrm{RBF}$ \\
\hline Parameter & $\sigma=200$ & $\sigma=500$ & $\sigma=500$ \\
\hline $\begin{array}{c}\text { Regularization } \\
\text { constant C }\end{array}$ & 5000 & 5000 & 5000 \\
\hline \begin{tabular}{c}
$\varepsilon$-insensitivity \\
\hline $\begin{array}{c}\text { Support } \\
\text { vectors } \\
\text { number }\end{array}$
\end{tabular} & $8(13.3 \%)$ & 3 & 8 \\
\hline $\begin{array}{c}\text { Estimation } \\
\text { error }\end{array}$ & 171 & 5 & $8(13.3 \%)$ \\
\hline
\end{tabular}

Table 2: Comparison of the estimation errors (MSE) between using SVR and using linear regression method

\begin{tabular}{|c|c|c|c|}
\hline Relation & $\begin{array}{c}\mathrm{CO} \% \mathrm{Vs} \\
\dot{V} \mathrm{O}_{2} \%\end{array}$ & $\begin{array}{c}\mathrm{SV} \% \mathrm{Vs} \\
\dot{V} \mathrm{O}_{2} \%\end{array}$ & $\begin{array}{c}\mathrm{TPR} \% \mathrm{Vs} \\
\dot{V} \mathrm{O}_{2} \%\end{array}$ \\
\hline SVR & 171 & 5 & 30 \\
\hline LS & 418 & 15 & 151 \\
\hline
\end{tabular}

Table3: Comparison of the model fitting errors (MSE) using SVR and linear regression methods $(\mathrm{N}=5)$.

\begin{tabular}{|c|c|c|c|}
\hline Relation & $\begin{array}{c}\mathrm{CO} \% \mathrm{Vs} \\
\dot{V} O_{2} \%\end{array}$ & $\begin{array}{c}\mathrm{SV} \% \mathrm{Vs} \\
\dot{V} O_{2} \%\end{array}$ & $\begin{array}{c}\mathrm{TPR} \% \\
\mathrm{Vs} \dot{V} \mathrm{O}_{2} \%\end{array}$ \\
\hline $\begin{array}{c}\text { SVR testing } \\
\text { error }\end{array}$ & $245 \pm 15$ & $8 \pm 2$ & $36 \pm 5$ \\
\hline $\begin{array}{c}\text { LS Testing } \\
\text { error }\end{array}$ & $521 \pm 19$ & $22 \pm 7$ & $130 \pm 12$ \\
\hline
\end{tabular}

\section{CONCLUSIONS}

This is the first time that SVR has been applied to experimentally investigate the steady state relationships between key central cardiovascular variables and oxygen consumption during incremental exercise. The impressive results obtained prove that SVR is an effective approach that can be recommended for the modeling of physiological data.

\section{ACKNOWLEDGEMENTS}

The authors acknowledge project DP0452186/2003 funded by ARC (Australian Research Council).

\section{REFERENCES}

Allor KM, Pivarnik JM, Sam LJ et al (2000) Treadmill economy in girls and women matched for height and weight. J. Appl. Physiol 89: 512-516.

Astrand PO, Cuddy TE, Saltin, B et al (1964) Cardiac output during submaximal and maximal work. J. Appl. Physiol 9: 268-274.

Beck KC, Randolph LN, Bailey KR et al (2006) Relationship between cardiac output and oxygen consumption during upright cycle exercise in healthy humans. J. Appl. Physiol 101: $1474-1480$.

Drucker H, Burges C, Kaufman L et al (1997) Support vector regression machines. In: Advances in Neural Information Procession Systems, edited by M. Mozer, M. Jordan \& T. Petsche. Cambridge, MA. p. 155-161.

Fairbarn MS, Blackie SP, McElvaney NG et al (1994) Prediction of heart rate and oxygen uptake during incremental and maximal exercise in healthy adults. Chest 105: 1365-1369.

Freedman ME, Snider GL, Brostoff P et al (1955) Effects of training on response of cardiac output to muscular exercise in athletes. J. Appl. Physiol 8:37-47.

Girosi F (1998) An equivalence between sparse approximation and support vector machines. Neural Computation, 20, 1455-1480.

Gunn SR (1997) Support Vector Macines for classificationa and regression, Technical Report, Department of Electronics and Computer Science, University of Southampton.

Knobloch K, Lichtenberg A (2005) Winterhalter M, Rossner D, Pichlmaier M, and Philips R. Noninvasive cardiac output determination by twodimensional independent Doppler during and after cardiac surgery. Ann Thorac Surg 80: 1479-1483.

Kobayashi Y, Andoh Y, Fujinami T et al (1978) Impedance cardiography for estimating cardiac output during submaximal and maximal work. J. Appl. Physiol 45: 459-462.

Reeves JT, Grover RF, Blount SG Jr et al (1961) Cardiac output response to standing and treadmill walking. $J$. Appl. Physiol 16: 283-288.

Reeves JT, Grover RF, Filley GF et al (1961) Circulatory changes in man during mild supine exercise. J. Appl. Phsiol 16: 279-282.

Richard R, Lonsdorfer-wolf E, Dufour S et al (2004) Cardiac output and oxygen release during vintensity exercise performed until exhaustion. Eur $J$ Appl Physiol 93: 9-18.

Rowell LB. Circulatory adjustments to dynamic exercise (1986) In: Human circulation regulation during physical stress. New York: Oxford University Press, p. 213-256.

Rowland T, Popoeski, Ferrone L (1997) Cardiac response to maximal upright cycle exercise in healthy boys and men. Med Sci Sport Exer 29:1146-1151.

Su SW, Wang L, Celler BG (2006), Estimation of Oxygen Consumption for Moderate Exercises by Using a Hammerstein Model, In Proceeding of the 28th Annual International Conference of the IEEE 
Engineering in Medicine and Biology Society, New York, USA, September, pp. 3427-3430.

$\mathrm{Su}$ SW, Wang L, Celler BG, Savkin AV, and Guo Y (2007). Identification and Control for Heart Rate Regulation during Treadmill Exercise, IEEE Transactions on Biomedical Engineering 54 (7): 12381246.

Su SW, Wang L, Celler B et al (2005) Estimation of walking energy expenditure by using support vector regression. In Proceedings of the 27th Annual International Conference of the IEEE Engineering in Medicine and Biology Society (EMBS), pp. 35263529, Shanghai, China.

Turley KR, Wilmore JH (1997) Cardiovascular responses to treadmill and cycle ergometer exercise in children and adults. J. Appl. Physiol 83: 948-957.

Valerity VG, Supriya BG (2003) Volatility forecasting from multiscale and high-dimensional market data. Neurocomputing, 55, 285-305.

Vapnik V (1998) Statistical learning theory. New York: Wiley. 